\title{
Some division theorems for vector fields
}

\author{
by ANDRZEJ ZaJTz (Kraków)
}

\begin{abstract}
This paper is concerned with the problem of divisibility of vector fields with respect to the Lie bracket $[X, Y]$. We deal with the local divisibility. The methods used are based on various estimates, in particular those concerning prolongations of dynamical systems. A generalization to polynomials of the adjoint operator $\operatorname{ad}(X)$ is given.
\end{abstract}

0. Introduction. The Lie bracket of differentiable vector fields on a smooth manifold is one of the fundamental operations not only in differential geometry. We deal with the following problem of division:

Given vector fields $X, Z$, does there exist a vector field $Y$ such that $[X, Y]=Z$ ?

The problem has been considered only for local vector fields and the full and positive answer is known whenever $X$ has a nonvanishing germ. In this case $X$ has local representation $\partial / \partial x_{1}$ and the "quotient" $Y$ can be taken to be

$$
Y\left(x_{1}, \ldots, x_{n}\right)=\int_{-\delta}^{x_{1}} Z\left(t, x_{2}, \ldots, x_{n}\right) d t
$$

for $\|x\|=\sup \left|x_{i}\right| \leq \delta$. This fact has been broadly exploited in papers concerning the well-known Pursell-Shanks theorem and its generalizations.

Since our problem will also be of local character it can be assumed that $X$ and $Z$ are vector fields defined in a neighbourhood of the origin 0 of $\mathbb{R}^{n}$ and the equality $[X, Y]=Z$ is meant in the sense of germs, that is, there exists a neighbourhood $U$ of 0 in which it holds.

Thus $X, Y, Z$ will be elements of the Lie algebra $\mathfrak{X}\left(\mathbb{R}^{n}\right)$ of local $C^{\infty}$ vector fields defined near the origin of $\mathbb{R}^{n}$. In view of the above, the question remains open only for homogeneous vector fields $X, Z$, that is, with $X(0)=$ $Z(0)=0$. From now on the notation $\mathfrak{X}\left(\mathbb{R}^{n}\right)$ will be used for the subalgebra of homogeneous elements.

1991 Mathematics Subject Classification: Primary 17B66.

Key words and phrases: Lie bracket of vector fields, bounds to flow and its prolongations. 
In order to justify what we deal with in the section that follows, let us see how the flow $\Psi_{t}$ of a given vector field $X$ can be involved in the problem of divisibility by $X$.

For any field $Z$ the transfer of $Z$ along the trajectories of $X$ is defined by

$$
\left(\Psi_{t}\right)_{*} Z=\left(D \Psi_{-t} \circ \Psi_{t}\right) Z \circ \Psi_{t},
$$

i.e.

$$
\left(\Psi_{t}\right)_{*} Z(x)=D \Psi_{-t}\left(\Psi_{t}(x)\right) Z\left(\Psi_{t}(x)\right) .
$$

The Lie bracket $[Z, X]$ is just the infinitesimal version of that and we have

$$
[Z, X]=\left.\frac{d}{d t}\right|_{t=0}\left(\Psi_{t}\right)_{*} Z .
$$

More generally,

$$
\frac{d}{d t}\left(\Psi_{t}\right)_{*} Z=\left[\left(\Psi_{t}\right)_{*} Z, X\right]
$$

Setting $Y_{t}=\left(\Psi_{t}\right)_{*} Z$ we can write

$$
Y_{t}^{\prime}=\left[Y_{t}, X\right] \quad\left(Y^{\prime}=\frac{d}{d t} Y\right) .
$$

This gives

$$
Z=-\left[\int_{0}^{t} Y_{s} d s, X\right]+Y_{t}
$$

since $Y_{0}=Z$. Without loss of generality we can assume that $X$ is complete so the range of $t$ is $(-\infty, \infty)$. If necessary we can replace $X$ by $f X$ where $f$ is a $C^{\infty}$ function which is 1 in a neighbourhood of 0 and has a compact support in the set where $X$ is defined. Suppose that

$1^{\mathrm{o}} Y_{t} \rightarrow 0$ as $t \rightarrow \infty$,

$2^{\circ}$ the integral

$$
Y(x)=\int_{0}^{\infty} Y_{t}(x) d t
$$

is convergent and $Y$ is $C^{\infty}$ in a neighbourhood of 0 . Then $Z=[X, Y]$ so $Y$ is a solution to the problem.

Since $X(0)=0$ we have $\Psi_{t}(0)=0$ for all $t$. If $x=0$ is asymptotically stable then $\Psi_{t}(x) \rightarrow 0$ as $t \rightarrow \infty$ for small $\|x\|$. As also $Z(0)=0$, it follows that $Z\left(\Psi_{t}(x)\right) \rightarrow 0$, but what we need is that $D \Psi_{-t}\left(\Psi_{t}(x)\right) Z\left(\Psi_{t}(x)\right)$ and all its $x$-derivatives converge to 0 as $t \rightarrow \infty$, uniformly in $x$. The study of this question will be the subject of the next section. 
1. Some bounds to flows. Consider the system of differential equations

$$
x^{\prime}=X(x) \quad\left(x^{\prime}=d x / d t\right)
$$

where $x, X(x)$ are $n$-vectors, $X$ is $C^{\infty}$ in a neighbourhood of $x=0$ and $X(0)=0$. Thus $X$ can be written

$$
X(x)=A x+h(x)
$$

with $A=D X(0)$ and $\|h(x)\| \leq L\|x\|^{2}$. We may assume that the Lipschitz constant $L$ is global, so that solutions to (1.1) are defined globally.

Assume that all the eigenvalues $\lambda_{i}$ of $A$ satisfy $\operatorname{Re} \lambda_{i}<0$ for $i=1, \ldots, n$ (for short: $\operatorname{Re} \lambda<0$ ). It is known that under this condition there exist positive constants $K$ and $c$ such that

$$
\left\|e^{t A}\right\| \leq K e^{-c t} \quad \text { for } t>0
$$

and $\delta>0$ such that

$$
\left\|\Psi_{t}(x)\right\| \leq K e^{-c t / 2}\|x\| \quad \text { for }\|x\| \leq \delta .
$$

Here $\Psi_{t}(x)$ is the solution of (1.1) passing through $x$ at $t=0$ (the flow of $X$ ). For the constant $c$ we may take any number $<\min (|\operatorname{Re} \lambda|$ ) (this is easily seen by writing $A$ in Jordan canonical form).

LEMMA 1.1. If there is a bound

$$
\left\|\Psi_{t}(x)\right\| \leq K e^{c(t)}\|x\| \quad \text { for }\|x\| \leq \delta, t \geq 0,
$$

with $K$ a constant and $c(t)$ depending only on the eigenvalues of $A$ and not on their multiplicities (as in the above case), then the derivatives $D^{k} \Psi_{t}(x)$, $k=1,2, \ldots$, also have similar bounds with the same $\delta$ and $c(t)$ and different constants $K_{k}$.

P r o o f. Consider the following variational equation $(k$ th prolongation of (1.1) with respect to $x)$ :

$$
\left\{\begin{array}{l}
x^{\prime}=X(x) \\
\xi_{1}^{\prime}=D X(x) \xi_{1} \\
\xi_{2}^{\prime}=D^{2} X \xi_{1} \xi_{1}+D X \xi_{2}, \\
\cdots \\
\xi_{k}^{\prime}=\sum_{s=1}^{k} D^{s} X \sum_{\substack{\alpha_{1}+\ldots+\alpha_{s}=k \\
\alpha_{i}>0}} \xi_{\alpha_{1}} \ldots \xi_{\alpha_{s}},
\end{array}\right.
$$

with $\xi_{\alpha} \in \mathbb{R}^{n}$ for $\alpha=1, \ldots, k$. With brief notation $\left(x, \xi_{1}^{\prime}, \ldots, \xi_{k}^{\prime}\right)=$ $F\left(x, \xi_{1}, \ldots, \xi_{k}\right)$ the Hessian of this equation, i.e. $D F(0)$, is of the form

$$
\left.\left(\begin{array}{llll}
A & & & \\
& A & & \\
& & \ddots & \\
& & & A
\end{array}\right) \quad \text { (of dimension }(k+1) n\right) \text {. }
$$


Thus $D F(0)$ has the same eigenvalues as $A$.

For any constant vector $v \in \mathbb{R}^{n}$ the system

$$
\left(\Psi_{t}(x), D \Psi_{t}(x) v, \ldots, D^{k} \Psi_{t}(x) v^{k}\right)
$$

is a solution to (1.3) passing through $(x, v, 0, \ldots, 0) \in \mathbb{R}^{(k+1) n}$. In fact,

$$
\begin{aligned}
\left(D^{k} \Psi_{t} v^{k}\right)^{\prime} & =\left(D^{k} \Psi_{t}\right)^{\prime} v^{k}=D^{k} \Psi_{t}^{\prime} v^{k}=D^{k}\left(X \circ \Psi_{t}\right) v^{k} \\
& =\left(\sum_{s=1}^{k} D^{s}(X) \Psi_{t} \sum_{\substack{\alpha_{1}+\ldots+\alpha_{s}=k \\
\alpha_{i}>0}} D^{\alpha_{1}} \Psi_{t} \ldots D^{\alpha_{s}} \Psi_{t}\right) v^{k}
\end{aligned}
$$

and $\left(D^{\alpha_{1}} \Psi_{t} \ldots D^{\alpha_{s}} \Psi_{t}\right) v^{k}=\left(D^{\alpha_{1}} \Psi_{t} v^{\alpha_{1}}\right) \ldots\left(D^{\alpha_{s}} \Psi_{t} v^{\alpha_{s}}\right)$. Therefore, if a bound $\left\|\Psi_{t}(x)\right\| \leq k e^{c(t)}\|x\|$ holds for $\|x\| \leq \delta$ and $t \geq 0$, then

$$
\left\|D^{l} \Psi_{t}(x) v^{l}\right\| \leq K_{l} e^{c(t)}\|(x, v, 0, \ldots, 0)\|, \quad l=1, \ldots, k,
$$

for all $\|x\| \leq \delta$ and any $\|v\| \leq 1$. This gives $\left\|D^{l} \Psi_{t}\right\| \leq K_{l}^{\prime} e^{c(t)}$.

LEMma 1.2. We have

$$
\left|\operatorname{det} D \Psi_{t}(x)\right| \geq M e^{(\operatorname{tr} A) t} \quad \text { for }\|x\| \leq \delta,
$$

with a positive constant $M$.

Pro of. Set $\Delta_{t}(x)=\operatorname{det} D \Psi_{t}(x)$. Then $\Delta_{t+s}(x)=\Delta_{t}\left(\Psi_{s}(x)\right)$. Hence

$$
\Delta_{s}^{\prime}(x)=\Delta_{0}^{\prime}\left(\Psi_{0}(x)\right) \Delta_{s}(x) .
$$

A routine computation leads to $\Delta_{0}^{\prime}(\xi)=\operatorname{tr} D X(\xi)$ and finally

$$
\Delta_{s}(x)=\exp \int_{0}^{t} \operatorname{tr} D X\left(\Psi_{s}(x)\right) d s,
$$

since $\Delta_{0}(x)=1$. By applying $(1.1)^{\prime}$ this can be written as

$$
\Delta_{s}(x)=e^{(\operatorname{tr} A) t} \exp \int_{0}^{t} \operatorname{tr} D k\left(\Psi_{s}(x)\right) d s .
$$

Since

$$
\left|\operatorname{tr} D k\left(\Psi_{s}(x)\right)\right| \leq C\left\|\Psi_{s}(x)\right\|^{2} \leq C \delta K e^{-c t},
$$

the integral $\int_{0}^{t} \operatorname{tr} D k\left(\Psi_{s}(x)\right) d s$ is bounded from below by $-C \delta K / c$. Thus we can take $M=\exp (-C \delta K / c)$.

LEMmA 1.3. There are constants $K_{k}$ and $L_{k}$ such that

$$
\begin{gathered}
\left\|D^{k} \Psi_{t}(x)\right\| \leq K_{k} e^{-c t / 2} \\
\left\|D^{k}\left[D \Psi_{-t}\left(\Psi_{t}(x)\right)\right]\right\| \leq L_{k} e^{(k+1) a t},
\end{gathered}
$$

where $a=-\operatorname{tr} A-(n-1) c$ and $\|x\| \leq \delta$.

Pro of. The bounds (1.5) follow immediately from Lemma 1.1 with reference to (1.2). 
For (1.6), from the identity $\Psi_{-t}\left(\Psi_{t}(x)\right)=x$ it follows that $D \Psi_{-t}\left(\Psi_{t}(x)\right)$ is equal to the inverse matrix to $D \Psi_{t}(x)$. In view of (1.5) and Lemma 1.2 the elements of $\left(D \Psi_{t}(x)\right)^{-1}$ are majorized in absolute value by

$$
e^{(-\operatorname{tr} A-(n-1) c) t}\left(=e^{a t}\right)
$$

up to a constant multiplicative factor.

Now from $\left(D \Psi_{t}\right)^{-1} \circ D \Psi_{t}=I$ we get

$$
D\left(D \Psi_{t}\right)^{-1} D \Psi_{-t}+\left(D \Psi_{t}\right)^{-1} D^{2} \Psi_{t}=0,
$$

which gives

and (1.6) follows by induction.

$$
\left\|D\left(D \Psi_{t}\right)^{-1}\right\| \leq L_{1} e^{2 a t}
$$

We denote by $\mathfrak{X}_{m}\left(\mathbb{R}^{n}\right)$ the space of local vector fields, $m$-flat at 0 .

TheOrem 1.4. Suppose $X$ is as above and $Z \in \mathfrak{X}_{m}\left(\mathbb{R}^{n}\right)$. If $(k+1) a-$ $m c / 2<0$ then there exists a $C^{k}$ vector field $Y$ in a neighbourhood of 0 such that $[X, Y]=Z$.

Proof. $Z$ being $m$-flat satisfies $\left\|D^{k} Z(x)\right\| \leq M_{k}\|x\|^{m-k}$ for $0 \leq k \leq$ $m-1$ and it is bounded for $k \geq m$ when $\|x\| \leq \delta$. We have

$$
\left\|D^{k}\left(D \Psi_{-t}\left(\Psi_{t}(x)\right) Z\left(\Psi_{t}(x)\right)\right)\right\| \leq \sum_{r+s=k}\left\|D^{r}\left(D \Psi_{-t} \circ \Psi_{t}\right)\right\|\left\|D^{s}\left(Z \circ \Psi_{t}\right)\right\| .
$$

By (1.2) and (1.5)

$$
\left\|\left(D^{u} Z\right) \circ \Psi_{t}\right\| \dot{\leq} e^{-(m-u) c t / 2} \quad \text { for } u \leq m-1,
$$

and the left hand side is bounded for $u \geq m$. Here and below, $\dot{\leq}$ indicates that the bound holds up to a multiplicative constant.

As the other term in (1.7) is bounded by $e^{-u c t / 2}$ we get

$$
\left\|D^{s}\left(Z \circ \Psi_{t}\right)\right\| \dot{\leq} e^{-m c t / 2}
$$

for all $s$ since $u \geq m$. In view of (1.6)-(1.8) we have

$$
\left\|D^{k}\left(D \Psi_{-t} \circ \Psi_{t}\right) Z \circ \Psi_{t} \mid\right\| \dot{\leq} e^{((k+1) a-m c / 2) t} .
$$

Suppose $(k+1) a-m c / 2<0$ for a positive integer $k$. Then the integral

$$
F(X, Z)=\int_{0}^{\infty}\left(\Psi_{t}\right)_{*} Z d t \quad\left(=\int_{0}^{\infty}(\exp t X)_{*} Z d t\right)
$$

is a vector field of class $C^{k}$ in the ball $\|x\| \leq \delta$.

Since clearly $\left\|\left(\Psi_{t}\right)_{*} Z\right\| \rightarrow 0$ as $t \rightarrow \infty$, we get by $(0.1)$

$$
[X, F(X, Z)]=Z \text {, }
$$

which was to be proved. 
2. Divisibility by linear vector fields. Suppose $X=A x$. Then $\Psi_{t}(x)=e^{t A} x$. As previously we assume that $A$ satisfies $\operatorname{Re} \lambda<0$. Then

Let $c$ be any constant $<\min (|\operatorname{Re} \lambda|)$ and $b$ any constant $>\max (|\operatorname{Re} \lambda|)$.

$$
\left\|e^{t a}\right\| \leq K e^{-c t}, \quad\left\|e^{-t A}\right\| \leq L e^{b t}, \quad t \geq 0
$$

We call

$$
d(X)=\frac{\max (|\operatorname{Re} \lambda|)}{\min (|\operatorname{Re} \lambda|)}
$$

the dispersion of $X$. Obviously $b / c>d(X)$.

TheOREM 2.1. Suppose that $Z$ is $m$-flat at $x=0$ and $m \geq d(X)+1$. Then $Z$ is divisible by $X$ with a quotient $F(X, Z)$ defined by (1.9).

Proof. Now

$$
\left(\Psi_{t}\right)_{*} Z(x)=e^{-t A} Z\left(e^{t A}\right)
$$

and

$$
D^{k}\left(\left(\Psi_{t}\right)_{*} Z(x)=e^{-t A} D^{k} Z\left(e^{t A}\right) e^{k t A}, \quad k \geq 0 .\right.
$$

Exploiting the $m$-flatness of $Z$ as in the proof of Theorem 1.4 we come to the following estimate:

$$
\left\|D^{k}\left(\left(\Psi_{t}\right)_{*} Z(x)\right)\right\| \dot{\leq} e^{(b-m c) t}, \quad k=0,1, \ldots
$$

The constants $b$ and $c$ may be taken such that $b / c<d(X)+1$. It follows that $b-m c<0$ for $m \geq d(X)+1$. Consequently, the integral (1.9) converges uniformly together with all its derivatives. Thus $F(X, Z)$ is a $C^{\infty}$ vector field in any ball contained in the domain of $Z$.

In particular, if $X=x$ then $d(X)=1$ and taking $m=2$ we conclude from Theorem 2.1 that each $Z$ from $\mathfrak{X}_{2}\left(\mathbb{R}^{n}\right)$ is divisible by $X$.

3. Divisibility by means of linearization. Consider again the general case $X=A x+h(x)$ as in (1.1). The field $X_{0}=A x$ is called the linearization of $X$ at 0 . From now on the vector field $X$ will be thought of locally as the germ at 0 of a smooth map $X: \mathbb{R}^{n} \rightarrow \mathbb{R}^{n}$.

Suppose that $X$ is $C^{\infty}$-equivalent to its linearization $X_{0}$, that is, there exists a $C^{\infty}$-diffeomorphism $f$ of $\mathbb{R}^{n}$, with $f(0)=0$, such that $f_{*} X=X_{0}$ in a neighbourhood of 0 .

For a given $Z$ set $Z_{0}=f_{*} Z$ and assume that there is a $Y_{0}$ such that $Z_{0}=\left[Y_{0}, X_{0}\right]$. This means

$$
f_{*} Z=\left[Y_{0}, f_{*} X\right]=f_{*}\left[\left(f^{-1}\right)_{*} Y_{0}, X\right] .
$$

Hence $Z=[Y, X]$ with $Y=\left(f^{-1}\right)_{*} Y_{0}$, and we obtain

LEMMA 3.1. If $f_{*} Z$ is divisible by the linearization of $X$ then $Z$ is divisible by $X$. 
Note that the transformation $f_{*}$ does not change the order of flatness of $Z$.

Which (germs of) vector fields are linearizable? The answer is: almost all. This can be concluded from the following theorems of Sternberg:

Either of the conditions below implies that a vector field $X$ with $X(0)=0$ is $C^{\infty}$-equivalent to its linearization $D X(0) x$.

(i) Each eigenvalue $\lambda$ of $D X(0)$ satisfies $\operatorname{Re} \lambda<0$ and

$$
X(x)=D X(0) x+o\left(x^{\infty}\right) .
$$

(ii) Each eigenvalue $\lambda_{i}(i=1, \ldots, n)$ satisfies

$$
\lambda_{i} \neq m_{1} \lambda_{1}+\ldots+m_{n} \lambda_{n}
$$

whenever the $m_{j}$ are non-negative integers with $m_{1}+\ldots+m_{n} \geq 2([1],[2])$.

Combining these facts with our results of previous sections, via Lemma 3.1 , we come to the following conclusion.

TheOREM 3.2. Suppose that $X$ is a $C^{\infty}$ vector field and $D X(0)$ has all eigenvalues with negative real parts. If $X$ satisfies either (3.1) or (3.2) then every vector field $Z, m$-flat with $m \geq d(X)+1$, is $C^{\infty}$-divisible by $X$.

Sternberg's algebraic condition (3.2) is also directly involved in the problem of divisibility of vector fields. Namely, let

$$
\sum a_{\alpha}^{i} x^{\alpha}, \quad \sum b_{\alpha}^{i} x^{\alpha}, \quad \sum c_{\alpha}^{i} x^{\alpha}
$$

be the Taylor series at $x=0$ for $X, Y, Z$ respectively. The equality $[X, Y]=$ $Z$ gives

$$
\sum_{\substack{\alpha+\beta=\gamma \\ j=1, \ldots, n}} b_{\alpha+1_{j}}^{i} a_{\beta}^{j}-a_{\alpha+1_{j}}^{i} b_{\beta}^{j}=c_{\gamma}^{i}
$$

with $1_{j}$ standing for the multiindex $(0, \ldots, 0,1,0, \ldots, 0)$, where 1 is in the $j$ th place. For given coefficients $a$ and $c$ there is a purely algebraic problem of solvability of this equation with respect to the unknown coefficients $b$.

Let us take $X=\sum_{i=1}^{n} \lambda_{i} x_{i} \partial / \partial x_{i}$. Then the $\lambda_{i}$ are the eigenvalues of $D X(0)$.

Let $\alpha=\left(\alpha_{1}, \ldots, \alpha_{n}\right), \alpha_{1}+\ldots+\alpha_{n}=|\alpha|$. In this case all $a_{\alpha}^{i}$ in formula (3.3) vanish for $|\alpha| \geq 2$. Hence (3.3) is now

$$
\sum_{j}\left(\sum_{k} b_{\alpha+1_{j}-1_{k}} a_{k}^{j}-a_{j}^{i} b_{\alpha}^{j}\right)=c_{\alpha}^{i} .
$$

Since $a_{j}^{i}=\lambda_{i} \delta_{j}^{i}$ and the number of the indices $j$ is $\alpha_{j}$ we get

$$
\left(\sum_{j} \alpha_{j} \lambda_{j}-\lambda_{i}\right) b_{\alpha}^{i}=c_{\alpha}^{i}
$$


Suppose that $Z$ is $m$-flat and $c_{\alpha}^{i} \neq 0$ for $|\alpha| \geq m$; then for the existence of $Y$ such that $[X, Y]=Z$ it is necessary to have

$$
\lambda_{i} \neq \sum_{j} \alpha_{j} \lambda_{j}
$$

for any non-negative integers $\alpha_{1}, \ldots, \alpha_{n}$ satisfying $|\alpha| \geq m$. This is exactly Sternberg's condition for $m=2$ (in the regularity class $k=\infty$ ).

If $\lambda_{i}=\sum \alpha_{j} \lambda_{j}$ and $\operatorname{Re} \lambda<0$ (or $\left.\operatorname{Re} \lambda>0\right)$ then

$$
\left|\operatorname{Re} \lambda_{i}\right|=\sum \alpha_{j}\left|\operatorname{Re} \lambda_{j}\right| \geq|\alpha| \min \left(\left|\operatorname{Re} \lambda_{j}\right|\right) .
$$

This implies $|\alpha| \leq \max \left(\left|\operatorname{Re} \lambda_{j}\right|\right) / \min \left(\left|\operatorname{Re} \lambda_{j}\right|\right)=d(X)$. Thus for $m \geq d(X)$ +1 we have $|\alpha| \leq m-1$ and the condition (3.4) is satisfied (as it should be in view of Theorem 3.2). This also shows that the lower bound $d(X)+1$ for $m$ in Theorem 3.2 is sharp.

On the other hand, if there are both negative and positive numbers in $\operatorname{Re} \lambda$ then the equality $\lambda_{i}=\sum \alpha_{j} \lambda_{j}$ may occur for all $|\alpha|$.

4. Generalization to polynomials. For some applications to actions of infinite Lie groups it is useful to know when polynomials of the adjoint mapping $\operatorname{ad}(X)$ act surjectively in the space of infinitely flat vector fields. An answer to this question is given in the following:

THEOREM 4.1. Let $P(\xi)=a_{0}+a_{1}+\ldots+a_{r} \xi^{r}$ be a polynomial of degree $r>0$. Suppose that $X$ satisfies $\operatorname{Re} \lambda<0$. For any vector field $Z$ vanishing up to infinite order at $x=0$ there exists a vector field $Y$ such that $Z=$ $P(\operatorname{ad}(X)) Y$. The $Y$ can be defined by

$$
Y(x)=-\int_{0}^{\infty} f(t)\left(\Psi_{t}\right)_{*} Z(x) d t
$$

where $f(t)$ is the solution of the differential equation

$$
a_{0} \xi-a_{1} \xi^{\prime}+\sum_{k=2}^{r}(k-1) a_{k} \xi^{(k)}=0,
$$

with initial conditions $\xi(0)=\ldots=\xi^{(r-2)}(0)=0, \xi^{(r-1)}(0)=1 /\left((r-1) a_{r}\right)$ for $r \geq 2$ and $\xi(0)=-1 / a_{1}$ for $r=1$.

P r o of. Equation (4.2) being with constant coefficients, there are positive constants $\alpha, \beta$ such that

$$
|f(t)| \leq \alpha e^{\beta t} \quad \text { for } t \geq 0 .
$$

As in Section 1, we have the following bounds:

$$
\left\|f(t) D^{k}\left(\Psi_{t}\right)_{*} Z(x)\right\| \leq \alpha M_{m}^{k} e^{\left(\beta+\gamma_{k}-m c\right) t}, \quad c>0
$$


for $t \geq 0$ and $\|x\| \leq \delta$. With $k$ fixed we can choose $m$ great enough so that $\beta+\gamma_{k}-m c<0$. This makes the integral (4.1) uniformly convergent in $B(\delta)$ together with all derivatives. Thus $Y$ is $C^{\infty}$ in $B(\delta)$.

Set $Y_{t}=\left(\Psi_{t}\right)_{*} Z$. In the introduction we saw that $Y_{t}^{\prime}=\operatorname{ad}(X) Y_{t}$. Hence

$$
Y_{t}^{(k)}=[\operatorname{ad}(X)]^{k} Y_{t}, \quad k \geq 1 .
$$

Therefore

$$
P(\operatorname{ad}(X)) f Y_{t}=a_{0} f Y_{t}+a_{1} f Y_{t}^{\prime}+\ldots+a_{r} f Y_{t}^{(r)} .
$$

From

$$
\left(f Y_{t}\right)^{(k)}=f Y_{t}^{(k)}+k\left(f^{\prime} Y_{t}\right)^{(k-1)}+(1-k) f^{(k)} Y_{t}
$$

for $k \geq 1$, we get

$$
f Y_{t}^{(k)}=\left(f Y_{t}\right)^{(k)}-k\left(f^{\prime} Y_{t}\right)^{(k-1)}+(k-1) f^{(k)} Y_{t} .
$$

On inserting this into (4.5) one gets for $r \geq 2$

$$
\begin{aligned}
P(\operatorname{ad}(X)) f Y_{t}= & \left(a_{0} f-a_{1} f^{\prime}+\sum_{k=2}^{r} a_{k}(k-1) f^{(k)}\right) Y_{t}+a_{1}\left(f Y_{t}\right)^{\prime} \\
& +\sum_{k=2}^{r} a_{k}\left[\left(f Y_{t}\right)^{(k)}-k\left(f^{\prime} Y_{t}\right)^{(k-1)}\right] \\
= & a_{1}\left(f Y_{t}\right)^{\prime}+\sum_{k=2}^{r} a_{k}\left[\left(f Y_{t}\right)^{(k)}-k\left(f^{\prime} Y_{t}\right)^{(k-1)}\right]
\end{aligned}
$$

according to our assumption on $f$. Now, by integrating either side with respect to $t$ over the interval $(0, \infty)$ and using notation (4.1) we obtain

$$
P(\operatorname{ad}(X)) Y=\left.a_{1} f Y_{t}\right|_{0} ^{\infty}+\left\{\sum_{k=1}^{r} a_{k}\left[\left(f Y_{t}\right)^{(k-1)}-k\left(f^{\prime} Y_{t}\right)^{(k-2)}\right]\right\}_{0}^{\infty}
$$

and $f$ satisfies $f(0)=f^{\prime}(0)=\ldots=f^{(r-2)}(0)=0, f^{(r-1)}(0)=1 /\left((r-1) a_{r}\right)$. So, in view of (4.4) for $k=0$, we have

$$
\left.f Y_{t}\right|_{0} ^{\infty}=-f(0) Y_{0}=-f(0) Z=0 .
$$

As the bound (4.3) can be extended to all derivatives of $f$ and the operator $\operatorname{ad}(X)$ is bounded in $B(\delta)$, there is a constant $M$ such that

$$
\left\|f^{(p)} Y_{t}^{(q)}\right\| \leq \alpha e^{\beta t}\left\|\operatorname{ad}(X)^{q}\right\|\left\|Y_{t}\right\| \leq M e^{\left(\beta+\gamma_{0}-m c\right) t}, \quad p, q \geq 0,
$$

with $\beta+\gamma_{0}-m c<0$. Therefore

$$
I_{k}=\left[\left(f Y_{t}\right)^{(k-1)}-k\left(f^{\prime} Y_{t}\right)^{(k-2)}\right]_{0}^{\infty}=0
$$

for $2 \leq k \leq r-1$. For $k=r$

$$
I_{r}=-f^{(r-1)}(0) Y_{0}+r f^{(r-1)}(0) Y_{0}=(r-1) f^{(r-1)}(0) Z .
$$


Coming back to (4.6) we finally get

$$
P(\operatorname{ad}(X)) Y=a_{r}(r-1) f^{(r-1)}(0) Z=Z,
$$

as required.

For $r=1$, we take $f(0)=-1 / a_{1}$. Then

$$
P(\operatorname{ad}(X)) Y=\left.a_{1} f Y_{t}\right|_{0} ^{\infty}=-a_{1} f(0) Z=Z .
$$

In particular:

(i) If $P(u)=a+u$, then $f(t)=-e^{a t}$ and

$$
Y=\int_{0}^{\infty} e^{a t}\left(\Psi_{t}\right)_{*} Z d t .
$$

(ii) If $P(u)=u^{r}, r \geq 2$, then

$$
Y=\frac{1}{(r-1) !(r-1)} \int_{0}^{\infty} t^{r-1}\left(\Psi_{t}\right)_{*} Z d t .
$$

This $Y$ satisfies

$$
Z=[X, \ldots[X,[X, Y]]] \quad(r \text { commutators }) .
$$

As we see from the proof one can expect existence of a solution to the equation $P(\operatorname{ad}(X)) Y=Z$ also in the case where $Z$ vanishes at $x=0$ up to a finite order $m$. This would depend on the polynomial $P$ and the required regularity class of $Y$ which is to be defined by formula (4.1).

\section{References}

[1] E. Nelson, Topics in Dynamics, I. Flows, Princeton University Press, Princeton 1969.

[2] S. Sternberg, On the structure of local homeomorphisms of Euclidean n-space, II, Amer. J. Math. 80 (1958), 623-631.

JONTKOWA GÓRKA 15A

30-224 KRAKÓW, POLAND

Reçu par la Rédaction le 18.9.1990

Révisé le 12.11.1992 